\title{
The Future Directions In Evolving Wi-Fi: Technologies, Applications ANd Services
}

\author{
Sumant Ku Mohapatra, Ramya Ranjan Choudhury, Pravanjan Das \\ Department of Electronics \& Telecommunication Engineering, Trident Academy of \\ Technology, Bhubaneswar, Odisha, India
}

\begin{abstract}
New research directions will lead to fundamental changes in the design of future WiFi networks. However, with an explosion of wireless mobile applications and services, there are still some challenges on the spectrum crisis and high energy consumption. Wireless system designers have been facing the continuously increasing demand for high data rates and spectrum sharing required by new wireless applications and therefore have started research on future WiFi wireless technologies that are expected to be deployed beyond 2020. In this article we propose WiFi key technologies and there prospective: WiFi CERTIFIED ${ }^{T M}$ ac and Wi Fi CERTIFIED passpoint ${ }^{T M}$. Also represent super WiFi such as WiGig solution, White Fi, HetNets and Cognitive Fi. Future applications and services facing these potential technologies are also discussed.
\end{abstract}

\section{KEYWORDS}

WiFi, Super WiFi, HetNets, IEEE standards, Cognitive WiFi, WiGig

\section{INTRODUCTION}

WiFi is surrounding us. What technologies will define future WiFi ? Will key technologies related to WiFi creates road map towards its future or emerging super WiFi technologies extends? This paper focuses on new development alternatives that creates wireless access solutions means future of WiFi.

\section{WI-FI KEY TECHNOLOGIES AND PROSPECTIVES.}

\section{Tri-band WiFi}

The utilization of tri-band WiFi gets new wireless solutions. By this network topology the data rate increased to $5 \mathrm{Gbps}$. The aim of this solution is utilized in enterprise to get increased wireless speed, improved wireless access and increased network capacity by using the IEEE802.11ad or WiGig standard.

\section{Recent LiFi over WiFi}

The new technology Light Fi ( $\mathrm{LiFi})$ is LED switching on/of technique which transfers data at high speed. The speed of the on and of switching of the LED is converted in terms of data rates. The speed is $1 \mathrm{Gbits} / \mathrm{sec}$. which is 10 times faster than WiFi. 
International Journal of Next-Generation Networks (IJNGN) Vol.6, No.3, September 2014

\section{Advanced Enterprise WiFi}

The new wireless technologies for enterprise environments in 2014 will change the trends of enterprise employees. The different ways will advance in coming years such as:

- Adoption of 802.11ad which represents the upcoming change in the IEEE 802.11 protocol and increases the data speed into the Gigabit world.

- Expansion of cloud for small and medium sized enterprises.

- WiFi- based location analytics will help for different organization to improve the customers and user WiFi experience, to increase business intelligence and to improving the security policy.

- BY using advance Hot Spot 2.0 and pass point open services.

- To allow user to log in to the WiFi network by using their social credentials.

\section{WiFi CERTIFIED ${ }^{\mathrm{TM}}$ AC}

- The WiFi CERTIFIED 802.11ac can deliver data rate which is more than double of a 802.11 network.

- It supports only the $5 \mathrm{GHz}$ frequency, means signal has less range.

- It supports multiple antennas that is MIMO(multiple input multiple output).

- The important feature $802.11 \mathrm{ac}$ is beam forming that gets around the general $5 \mathrm{GHz}$ range problem. It States with 802.11 ac the signal is propagate for broadcast directly from the access point(AP) to a certain device and back towards the AP.

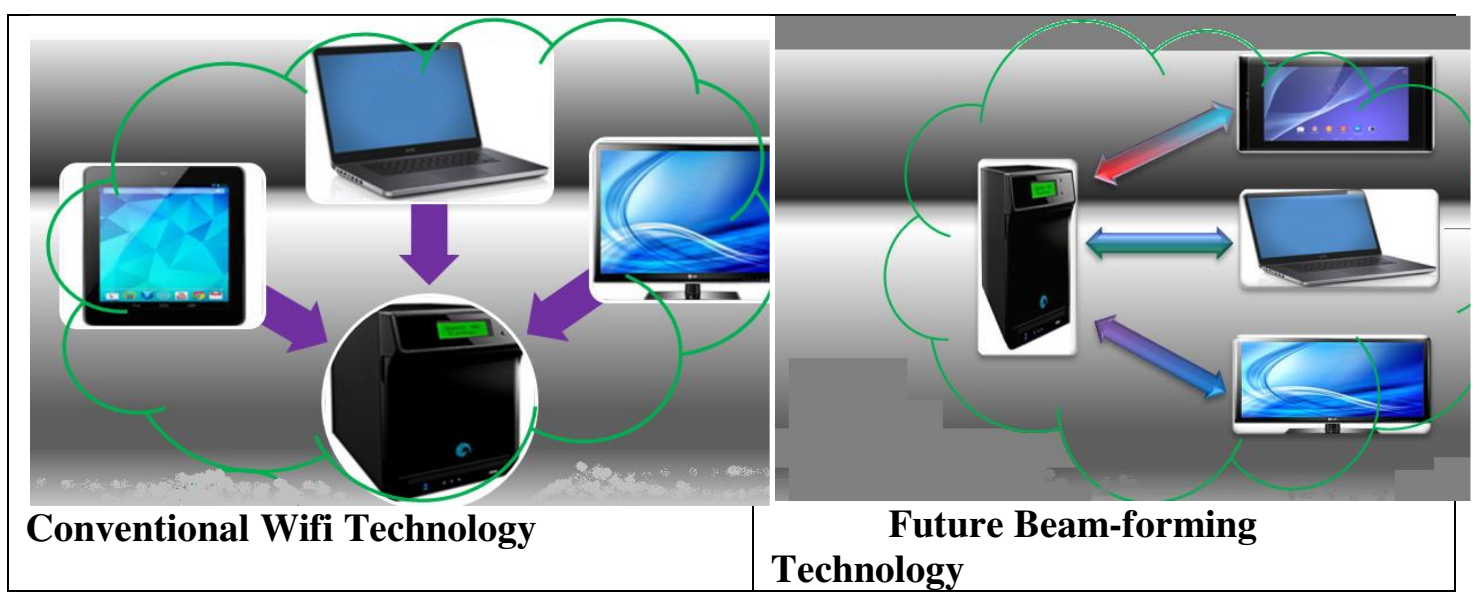

Figure 1.802.11ac Beam-forming technique compared with past conventional Wi-Fi

This future technique shown in Figure 1 as compared to conventional WiFi.WiFi certified equipments meets the security requirement of essential corporate networks and applications and allows residential users to protect their networks [7].

\section{Wi- FI CERTIFIED Passpoint ${ }^{\mathrm{TM}}$}

Wi- Fi Certified Passpoint ${ }^{\mathrm{TM}}$ has given the layout for foundation for latest type of Wi-Fi experience in public locations. Online sign-up for mobile devices without SIM card is the current passport release for this policy control.Wi- Fi Certified Passpoint ${ }^{\mathrm{TM}}$ ac builds up the collaboration of the passport, thereby attaining higher capacity of network and improving performance for 
International Journal of Next-Generation Networks (IJNGN) Vol.6, No.3, September 2014

multi-media content. The new streams of value addition for existing services of end-users is achieved through inter carrier WiFi roaming [2].

\section{Future WiFi speeds}

From global research [1] , in 2017 (20.3Mbps) the WiFi connection speeds generated from dual mode devices will increase 3 times to speeds in 2012(7.7Mbps). The Table-1 shows the projected WiFi network connection speeds in Kbps by Global region and different countries. The Figure-2 and 3 illustrates the highest growth in WiFi speeds from 2012 to 2017 with a CAGR (Compound Annual Growth Rate) in Percentage and expected Wi-Fi Network Connection Speeds by Global Regions from 2014- 2017 respectively[4].

Table-1 - Wi-Fi Network Connection Speeds by Global Regions from 2012- 2017 (in Mbps)

\begin{tabular}{|l|l|l|l|l|l|l|l|}
\hline Region/Year & 2012 & 2013 & 2014 & 2015 & 2016 & 2017 & CAGR \\
\hline $\begin{array}{l}\text { North } \\
\text { America }\end{array}$ & 11 & 14 & 16 & 18 & 21 & 23 & $15 \%$ \\
\hline $\begin{array}{l}\text { Latin } \\
\text { America }\end{array}$ & 5 & 6 & 7 & 8 & 9 & 11 & $18 \%$ \\
\hline $\begin{array}{l}\text { Western } \\
\text { Europe }\end{array}$ & 9 & 11 & 12 & 14 & 17 & 18 & $14 \%$ \\
\hline $\begin{array}{l}\text { Central \& } \\
\text { Eastern } \\
\text { Europe }\end{array}$ & 10 & 12 & 15 & 17 & 20 & 23 & $19 \%$ \\
\hline $\begin{array}{l}\text { Middle East } \\
\text { \& Africa }\end{array}$ & 3 & 3 & 3 & 4 & 4 & 4 & $8 \%$ \\
\hline Asia Pacific & 6 & 8 & 10 & 13 & 16 & 19 & $26 \%$ \\
\hline Global & 8 & 10 & 12 & 14 & 17 & 20 & $21 \%$ \\
\hline
\end{tabular}

Courtesy:- Cisco VNI 2013 Report

\section{International WiFi Roaming}

WiFi roaming is a vital business related advantages for operators. Now users expect WiFi to be available wherever and whenever they travel. Till now a revenue opportunity has not been successfully captured by operators.

\section{A. Super Wi-Fi : The Real Future}

To achieve todays spectrum demand different wireless access methods are utilized. Now-a-days WiFi has very much congestion and also provided the consumer a economical path to the network. To achieve the goal of affordable access to all consumers super WiFi is used. Super WiFi will be provided over radio spectrum to share among different user and internet service providers [8].

The major advantages of super Wi-Fi are :-

- Super Wi-Fi (400 meters) signals can travel over long distances than WiFi (100 meters). It can travel 4 times the Wi-Fi as a result more bandwidth, lower network costs and lower power consumption. 
International Journal of Next-Generation Networks (IJNGN) Vol.6, No.3, September 2014

- It can penetrate two or three concrete walls but Wi-Fi cannot.

- Greater efficiency due to longer distance coverage, wider range, more bandwidth and lower power consumption.

- Super Wi-Fi devices have the ability to switch from one group of channels to another.

- Advanced and powerful features.

- Self-explanatory and user friendly interface.

We use to define new development alternatives that create wireless access solutions in future, including:

- WiFi Gigabit Dolution (IEEE 802.11 ad standard)

- White Fi solution (IEEE 802.11 af standard)

- Wi-Fi approach for Machine to Machine (M2M) communication (IEEE 802.11 ah standard)

- HetNets solution

- Cognitive-Fi (CogFi)

\section{WI-FI GIGABIT SOLUTION: IEEE 802.11AD STANDARD}

The next generation beyond $802.11 \mathrm{ac}$ is IEEE 802.11 ad which is known as wireless Gigabit. It has the speed ability upto $7 \mathrm{Gbps}$ to our Wi-Fi network. It uses $60 \mathrm{GHz}$ frequency band instead of $5 \mathrm{GHz}$ and $2.4 \mathrm{GHz}$ band. The important drawback of Wi-Gig is the range which is very much shorter than IEEE 802.11ac standard Wi-Fi. The WiGig products are targeted to launch by the start of 2015. Figure - 4 shows Wi-Gig at 7Gbps wireless speed and related application areas[7].

\section{Wi-Gig Beam Management}

The antenna beam management is a key aspect of feature of Wi-Gig. The beam forming is obtained by using a bi-directional training sequence that is appended to each transmission. By this, system enables to transmit and /or the received beams to achieve the optimum link properties. This enables the system to overcome any movement of the transmitter, receiver or objects between them that might alter the path characterization.

\section{High Capacity}

It deploys 16-32 antenna element arrays which generates real spatial separation. For this it makes high capacity wireless deployments.

\section{Power Efficient}

It means multiple Gigabits per second of real throughput at only hundred of milli-watts of power of the total system. So it is a most power efficient technology in the Wi-Fi.

\section{WHITE FI SOLUTION: IEEE 802.11AF STANDARDS}

Super WiFi or TV white space refers to unused radio spectrum in TV broadcast bands typically at $700 \mathrm{MHz}$. This is an advance technology compared to Wi-Fi having a lot of extra spectrum. So it is termed as "White Fi". It does not face issues such as quality degradation and distance limitations as Wi-Fi. This is approved in February 2014.The proposal for the implementation of White Fi is still in its development stages. 
International Journal of Next-Generation Networks (IJNGN) Vol.6, No.3, September 2014

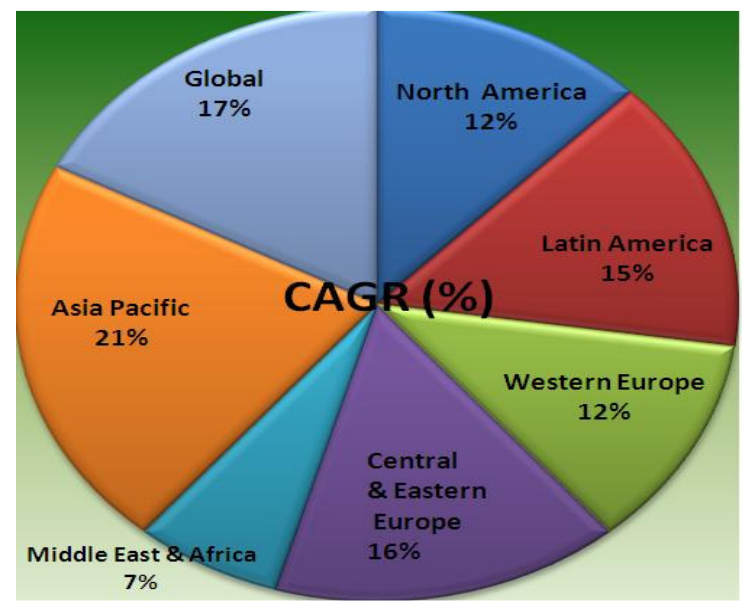

Figure 2. Plot illustrates the highest growth in WiFi speeds from 2012 to 2017 with CAGR in percentage

It provides a better effect to use more radio spectrum in an area where available bandwidth is at a premium. It also utilizes the resource more effectively. The TV channel spectrum occupied by white spaces, sometimes referred to as $700 \mathrm{MHz}$ beach front. Spectrum is ideal for penetrating trees, buildings and home walls just like TV. The same trees and structures block $900 \mathrm{MHz}$ and $2.4 \mathrm{GHz}$ Wi-Fi. The propagation path loss as well as the attenuation by materials is lower (in UHF and VHF bands) than conventional Wi-Fi standard.

\section{WI-FI APPROACH FOR MACHINE TO MACHINE (M2M) COMMUNICATION (IEEE 802.11 AH STANDARD)}

Its purpose is at creating large groups of stations that co-operate to share air- medium while minimizing energy consumption. It defines WiFi system operating at $1 \mathrm{GHz}$ (approximately). Final approval may be on March 2016.It provides improved transmission range compared to conventional Wi-Fi due to its favorable propagation characteristics.

Advanced applications include Large ScaleSensor networks, extended range hotspot and outdoor Wi-Fi for cellular traffic off-loading.[10]

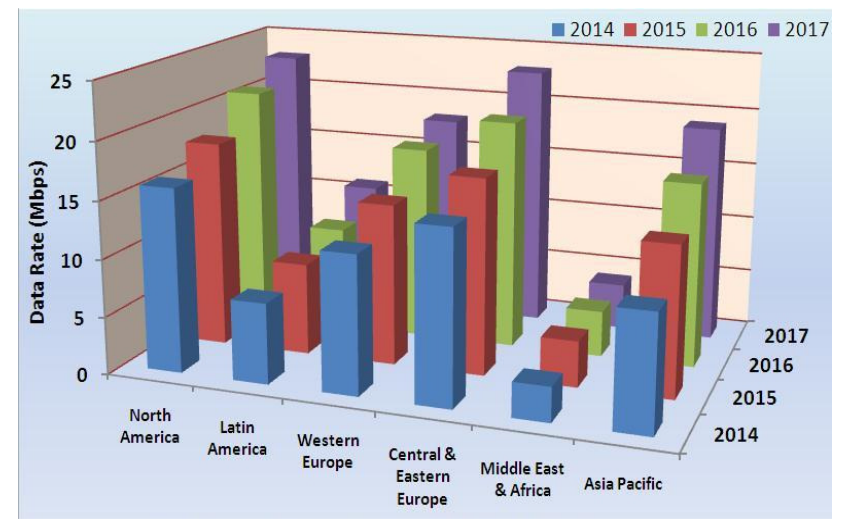

Figure 3. Expected Wi-Fi Network Connection Speeds by Global Regions from 2014- 2017 
International Journal of Next-Generation Networks (IJNGN) Vol.6, No.3, September 2014

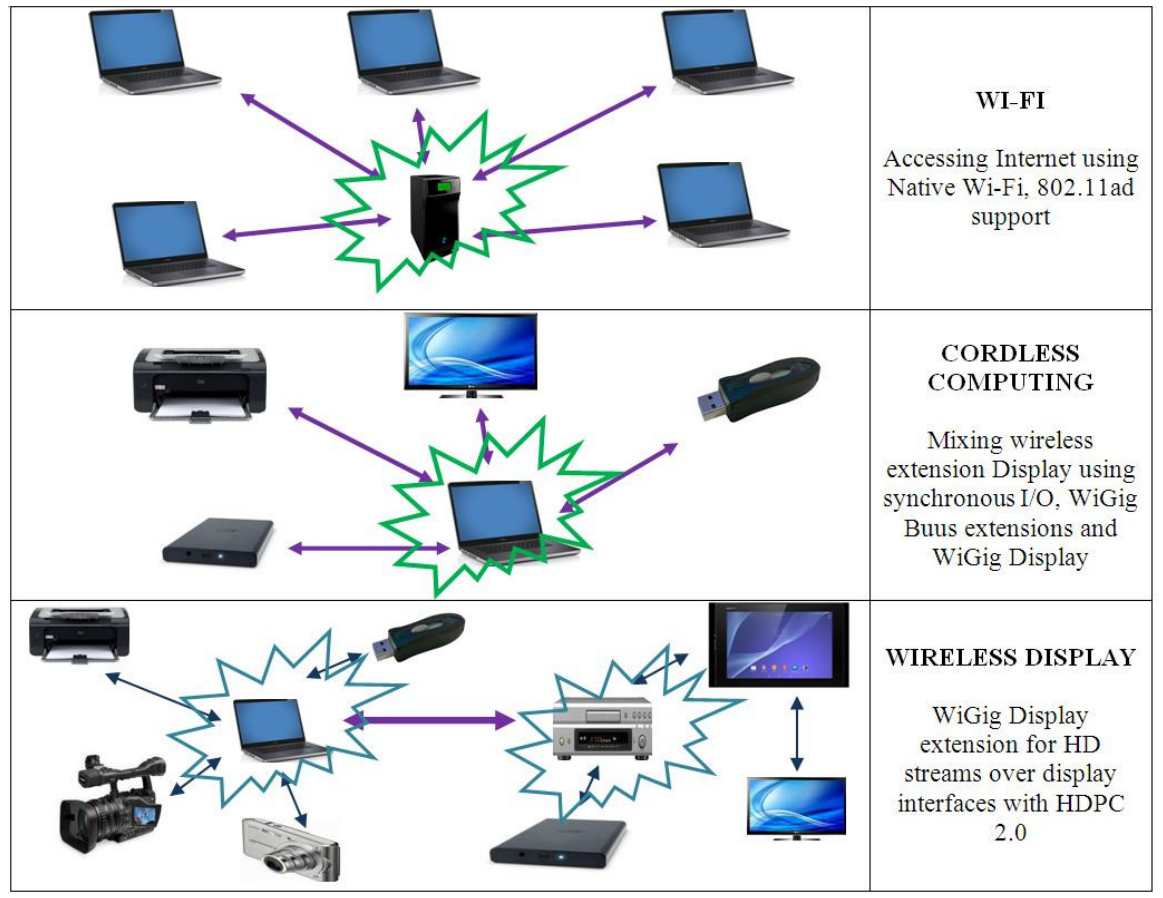

Figure 4.WiGig(7GbpsWiF) standard implemented in a wide range of applications.

M2M communications are projected to be one of the fastest growing technology segments of the IT sector in the coming years. In this, sensors and actuator networks connect communication machines and devices so that the automatically transmit information, serving the growing demand for environmental data acquisition.

\section{HETNETS SOLUTION}

When users have to be close to the base station small cells are required, but large cells are most cost effective to serve. So the best spectrum solution is deployed to overcome WiFi and Heterogeneous Network (HetNet). This is a mixed technology having different frequencies, cell sizes and network architectures to fulfill the rapid change of customer demand [3]. The services of HetNets are to analyze, plan, optimize and manage the best capacity upgradeplan including $\mathrm{Wi}-\mathrm{Fi}$, small cells and in-building solutions. The global heterogeneous network market is growing at $27.9 \%$ CAGR over the period of 2014-2018. The Key factor for this growth relates todemand for mobile broadband and the emergence of Wi-Fi enabled small cells. Customers want more from theirwireless providers, but current infra-structure cannot handle the growing traffic. Small cells and HetNets are expected to overcome some burden [5]. 


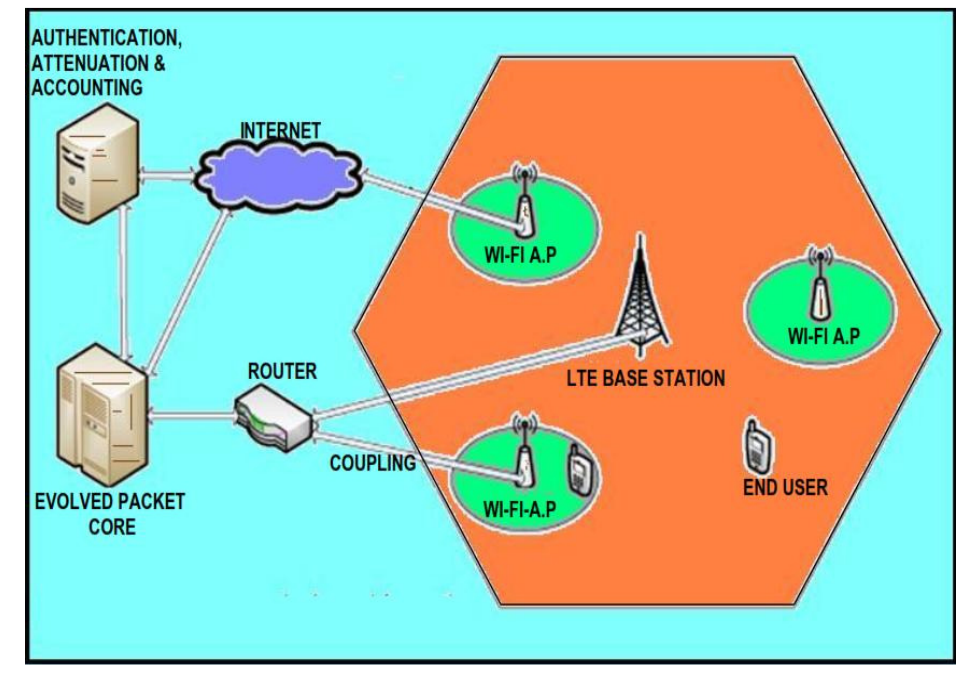

Fig 5:-WiFi Heterogeneous (HetNet) LTE-Network

An LTE-WiFiHetNet have a single base station (BS) and many number of WiFi access points (APs) can provide service to the same coverage area. Figure -5 shows such typical network architecture which provides a mixer of networks (Long Term Evolution and Wi-Fi network) implemented in hierarchical levels. The coupling elements are connected in between routers and $\mathrm{Wi}-\mathrm{Fi}$ access points as shown in figure 5. This junction of the network is packed tightly. There is another coupling element that connects internet access with WiFi access point. But this connection is loosely tied.

\section{COGNITIVE WIFI: Cog-FI}

Cognitive network and WiFi are two promising technique for efficient spectrum sharing. Now-adays the rapid change of consumer demand towards spectrum sharing overcomes by cognitive network rather than Wi-Fi. Currently wireless communications have been severely limited by the heterogeneity, which causes the transmission capacity and end-to-end performance for the wireless systems since it cannot be improved fundamentally. Therefore, in recent years the concept of cognitive network and Wi-Fi have been studied and discussed extensively from both academia and industrial communities [9]. To achieve this multiple interfaces such as internet, WiFi, Bluetooth and Zigbee are connected through cognitive network as shown in figure 6. 


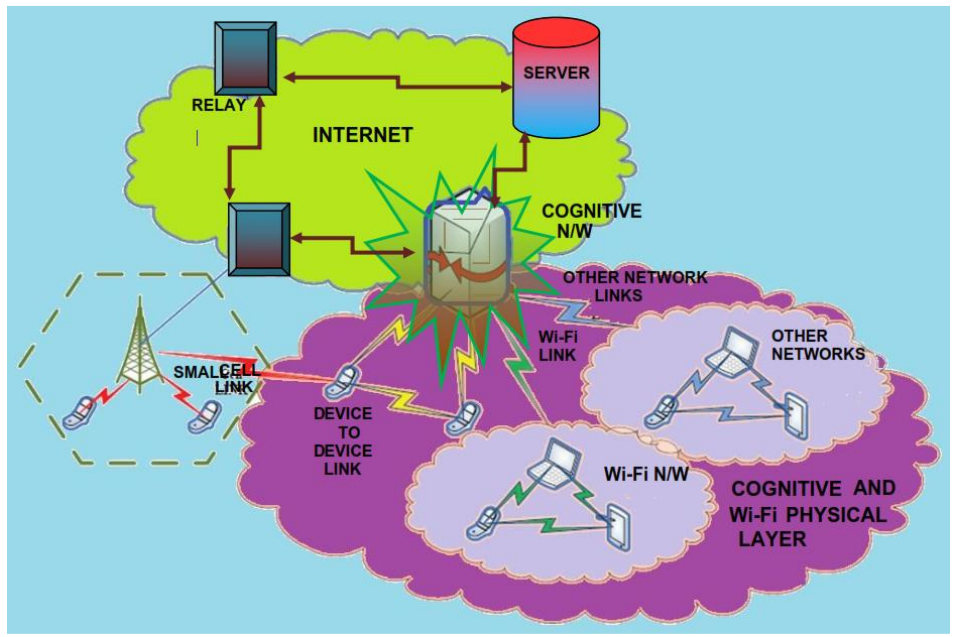

Figure 6-Connectivity Interfaces of Cognitive network with Wi-Fi

\section{RECENT APPLICATIONS AND SERVICES}

The UK communications regulatory has proposed a satellite system for aircraft and ships to provide WiFi connections. For this, the speed of Wi-Fi is ten times faster than conventional Wi-Fi used for public transport.Many service providers have planned to launch high speed Wi-Fi services that will provide internet service to customers at locations apart from their homes. They will be tying up with malls, coffee chains and food courts to provide their unlimited Wi-Fi services to its customers apart from their homes.[1]Stanford university researchers are trying for zippier Wi-Fi in crowded buildings by making a single, dense Wi-Fi infrastructure. For this each resident can use and manage its own private network.Recent developed Wi-Fi certified ac brings higher performance to enterprise networks by increasing its speed and capacity and also more efficient use of the wireless spectrum.Every fan in a packed sports stadium or music venue will soon be able to get bespoke content direct to their phones by location-aware Wi-Fi system developers by UK.US Navy leaders have planned to issue tablets in equipped ships and submarines with Wi-Fi. Sailors could use it to communicate at any point during the day.

Global public Wi-Fi hotspot numbers are set to grow from 1.3 million in 2011 to 5.8 million by 2015, marking a 350 percent increase according to research published by the Wireless Broadband(WBA).Recently launched WiFi Baby Camera by D-Link (India) offers a lot of great features to help a new form anywhere and also take away some of the stress related to child security and care.

\section{Wi-Fi in Hospitals}

Use of Wi-Fi technologies in hospitals grew rapidly day to day to create a future care to patients. Now-a-days voice over WiFi has enabled clinicians to co-ordinate care from anywhere in the hospital. Also Wi-Fi is beneficial to hospital staff, patients and their families and visitors to access the internet within the hospital. The application of WiFi in hospitals are classified as life critical, mission critical and consumer critical [2]. 
The hospitals use Wi-Fi technologies to:

- Smoothen then patient care and safety through automated sending of lab results to mobile phones.

- Increase efficiency with reliable, pervasive Wi-Fi coverage.

- Improving the patient satisfaction by allowing patients to connect with their family members and to enjoy the entertainment such as e-books, music and video.

- Enable video communication for caregivers to provide language interpretation services, education, poison control help and psychiatric services remotely.

- Make a co-ordination by sending alarms and alerts to people who are in closest proximity to deliver care.

- Provide real-time information on assets and patients through context aware services.

- Provide alarm and alert notification out to mobile clinicians and caregivers via their wireless phones.

\section{Conclusions}

This paper has discussed the future research directions that could lead to fundamental changes in the new development alternatives that create wireless access solution in future: WiGig, White-Fi, HetNets , M2M approach and cognitive WiFi. These solutions will form the basis of future WiFi. Recent developed applications and services designed a road map for future WiFi consumers. These applications place demands on available technology and spectrum in terms of both the capacity available and the speed that can be supported for multiple simultaneous users/uses.Higher speed and higher capacity Wi-Fi supported by additional spectrum will facilitate the continued development of wireless connected computing supporting high end-to-end connectivity speeds, improved quality of service in contested environments, higher mobile traffic offload and support the development of cloud computing applications. The future of Wi-Fi security will be very bright if we overcome the security problems in the present technologies such as modulation spectrum technique, default password settings, wireless equivalent privacy, service set identifier etc. And if we protect Wi-Fi from these security problems, we have long way to produce great technologies under Wi-Fi Networking in future. The security requirements vary depending on the amount of network traffic and the level of secrecy required for the information being exchanged and the application being used. Wi-Fi protected access is designed to meet these different requirements by running in two different modes, enterprise and home.Although the future is uncertain, the Wi-Fi industry will track record in innovation over the years. This has been first-rate and it shows no signs of slowing down now.

\section{REFERENCES}

[1] http://ciscovni.com/gist/index.html

[2] http://www.merunetworks.com

[3] Jeffery Andrews, "Seven ways that HetNets are a cellular paradigm Shift", IEEE Communication Magazine, Vol. 51 No.3 March. 2013.

[4] http://www.zdnet.com/super-fast-wi-fi-coming-802-11ac-2013-7000025352/

[5] Qualcomm, "A Comparison of LTE Advanced HetNetsand WiFi," white paper, WiFi.pdf,Sept. 2011.http://www.qualcomm.com/media/

[6] http://www.wi-fi.org/news-events/newsroom/

[7] Verma, L. ;Fakharzadeh, M. ; Sunghyun Choi, "Wifi on steroids: 802.11AC and 802.11AD ",Wireless Communications, IEEE Vol.20,Issue: 6, December 2013.

[8] HyoilKim ; Sch. of Electr. \&Comput. Eng., Ulsan Nat. Inst. of Sci. \& Technol. (UNIST), Ulsan, South Korea ;Jaehyuk Choi ; Shin, K.G., "Hierarchical Market Competition in a Duopoly Super Wi- 
International Journal of Next-Generation Networks (IJNGN) Vol.6, No.3, September 2014

Fi Spectrum Market ",Selected Areas in Communications, IEEE Journal,Vol-31-No.11, November 2013

[9] Bouet, M. ;Nguengang, G. ; Conan, V. ; Kousaridas, A. ; Spapis, P. ; Alonistioti, N.“Embedding cognition in wireless network management: an experimental perspective",Communications Magazine, IEEE,Vol. 50 ,Issue: 12,2012, Page(s): 150 - 160

[10] Bennis, M. ;Simsek, M. ; Czylwik, A. ; Saad, W. ; Valentin, S. ; Debbah, M.,"When cellular meets WiFi in wireless small cell networks", Communications Magazine, IEEE, Volume: 51 , Issue: 6,2013,Page(s): $44-50$.

\section{Authors}

Sumant Ku Mohapatra is working as Assistant Professor in Trident Academy of Technology, Bhubaneswar affiliated to B.P.U.T, Odisha, India. His research interests include wireless communication, digital signal processing, image processing and optical fiber communication

Ramya Ranjan Choudhury is working as Assistant Professor in Trident Academy of Technology; Bhubaneswar affiliated to B.P.U.T, Odisha, India. His areas of research interests are in satellite \& wireless communication, digital image processing and optical fiber communication

Pravanjan Das worked in Trident Academy of Technology, B.P.U.T, Bhubaneswar, Odisha, India as an Assistant Professor. His research interest isin wireless communication. Now he is working in Ericsson India Global Services Pvt. Ltd.
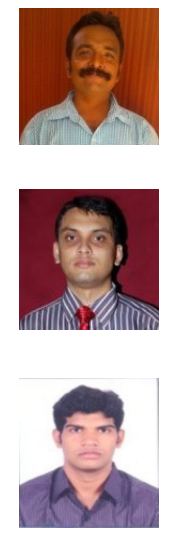